\title{
Comportamento de propriedades físicas, químicas e reológicas do suco de acerola armazenado a baixa temperatura ${ }^{1}$
}

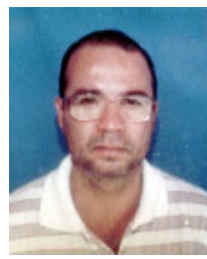

José E. Gomes², Dilermando Perecin ${ }^{3}$, Antônio B. G. Martins ${ }^{4}$ \& Sérgio R. Fontes ${ }^{5}$

1 Extraído da tese de Doutorado do primeiro autor, na FCAVJ/UNESP, financiada pela Fundação de Amparo à Pesquisa
do Estado de São Paulo (FAPESP)
2 Depto. Ciências Exatas da FCAVJ/ UNESP, Rod. Paulo Donato Castellani km 5, CEP 14.870-000, Jaboticabal, SP (Foto)
3 Depto. de Ciências Exatas da FCAVJ/UNESP. Telefax: (16) 323-2500, Ramal: 227. E-mail: perecin@.unesp.br
${ }^{4}$ Depto. de Horticultura da FCAVJ/UNESP. Telefax: (16) 323-2500, Ramal: 226. E-mail: baldo@fcav.unesp.br
5 Depto. de Ciências Exatas da FCAVJ/UNESP. Telefax: (16) 323-2500, Ramal: 112. E-mail: srfontes@fcav.unesp.br

Protocolo 137 - 14/11/1999

Resumo: Estudou-se o comportamento físico, químico e reológico do suco de cinco genótipos de acerola (CL2, CL5, CL11, R1 e R2) depois de lavadas, as frutas (2 kg) foram esmagadas em um processador manual. Do extrato obtido, em peneira de 1,6 mm, para separar o suco dos resíduos e sementes, foi acondicionado $20 \mathrm{~mL}$ de suco de cada amostra em tubos plásticos com dimensões $5 \times 3 \mathrm{~cm}$, que foram armazenados em freezer a uma temperatura de $-10{ }^{\circ} \mathrm{C}$, durante 10 meses. As leituras de sólidos solúveis totais foram refratométricas, o $\mathrm{pH}$ foi aferido através de um potenciômetro, o teor de vitamina $C$ pela técnica da redução do iodo e a viscosidade aparente com viscosímetro de cilindros concêntricos. As determinações foram realizadas inicialmente (mês 0) e bimestralmente $\left(2^{\circ}, 4^{\circ}, 6^{\circ}, 8^{\circ}\right.$ e $10^{\circ}$ mês). Os parâmetros de viscosidade e vitamina C foram os que mais variaram. O genótipo CL-2 se destacou no teor de sólidos solúveis totais e os $\mathrm{R} 1$ e R2 em teores de vitamina $\mathrm{C}$. Os sucos analisados mostraram comportamento reológico não-newtoniano e pseudoplástico.

Palavras-chave: Malpighia punicifolia, vitamina C, suco, viscosidade

\section{Physical, chemical and rheological behavior of West Indian cherry juice stored at low temperature}

\begin{abstract}
With the objective to study physical, chemical and rheological behavior of West Indian cherry juice of five genotypes (CL2, CL5, CL11, R1 and R2) an experiment was conducted with the juice extracted from $2 \mathrm{~kg}$ of mature fruits after washing, dried and squashed in a manual processor. The juice was sieved in a $1.6 \mathrm{~mm}$ sieve; $20 \mathrm{~mL}$ samples were packed in plastic tubes $(5 \times 3 \mathrm{~cm})$ and stored in a freezer at $-10{ }^{\circ} \mathrm{C}$ for a 10 month period. The evaluations of total soluble solids were refractometric, the $\mathrm{pH}$ was determined by potentiometer, vitamin $\mathrm{C}$ was measured by iodine reduction technique and apparent viscosity determined in concentric cylinders viscosimeter. The determinations were done initially and at intervals of two months $\left(0,2^{\text {nd }}, 4^{\text {th }}\right.$, $6^{\text {th }}, 8^{\text {th }}$ and $\left.10^{\text {th }}\right)$. Viscosity and vitamin $C$ were the characteristics which varied highly. The genotype CL-2 surpassed other genotypes in total soluble solids and the genotypes R1 and R2 showed superiority in vitamin $C$ content. The analyzed juices presented a non-newtonian and pseudoplastic rheological behavior.
\end{abstract}

Key words: Malpighia punicifolia, physical-chemical behavior, juice, viscosity, vitamin C

\section{INTRODUÇ̃̃O}

A acerola (Malpighia punicifolia) é uma das maiores fontes naturais de vitamina $\mathrm{C}$, condição que desperta interesse no mercado de frutas. Sua comercialização intensiva ocorre sob congelamento e, por ser um fruto perecível, a polpa congelada é a maneira mais prática de se atender aos consumidores, tendo em vista que a acerola disputa uma faixa de mercado, cujos consumidores preferem sucos naturais, com pouco processamento e características semelhantes ao "in natura".

A qualidade do suco congelado de acerola é resultado da manutenção de suas propriedades físico-químicas e da quantidade das substâncias componentes próximas às dos patamares do suco "in natura".

Nagy et al. (1993), descreveram que o comportamento reológico dos fluidos está dividido em newtonianos e não-newtonianos, sendo os newtonianos caracterizados por 
uma relação linear entre tensão de cisalhamento e a taxa de deformação aplicada, dependendo apenas da temperatura e da composição do fluido, enquanto os não-newtonianos são os fluidos inelásticos, dependentes ou independentes do tempo, de modo que, os independentes não são afetados pelo histórico anterior de cisalhamento, classificando-se como pseudoplásticos. Ibarz et al. (1996) salientaram a importância do conhecimento do comportamento reológico dos derivados de frutas, que deve ser utilizado nos parâmetros de qualidade, de avaliação e operação dos equipamentos processadores de alimentos.

Trifiró et al. (1987) relataram que sucos e purês de frutas, reologicamente, são fluidos pseudoplásticos e o afastamento do comportamento newtoniano é determinado pelo conteúdo de polpa, de modo que, sucos despolpados se comportam como newtonianos e o comportamento reológico se altera em função do conteúdo de polpa, por ação enzimática ou mesmo mecânica que modifiquem a estrutura da polpa. Por outro lado, Tanglertpaibul \& Rao (1987) reportaram que o comportamento reológico de sucos e purês de frutas está ligado aos teores de sólidos solúveis em suspensão em função da forma, tamanho, concentrações das partículas suspensas e da estrutura do sistema. As referências que tratam da reologia de derivados de frutas estabelecem que a temperatura, a concentração de sólidos solúveis, o teor de pectina e de sólidos insolúveis são os principais responsáveis pelo comportamento reológico (Queiroz, 1998).

O principal modelo reológico para "fluidos nãonewtonianos" é o modelo da Lei da Potência (Rao \& Anantheswaram, 1982) em que se considera a tensão inicial e é recomendado para fluidos que iniciam o processo de escoamento quando a tensão de cisalhamento aplicada supera a tensão inicial característica de cada um desses fluidos (Queiroz, 1998). São exemplos de modelos reológicos: modelos de Bingham; de Herschal-Bulkley; de Casson e de Mizrahi-Berk.

Holdsworth (1971) afirma que a maioria dos fluidos alimentícios apresenta comportamento pseudoplástico, cuja viscosidade aparente decresce com o aumento da taxa de deformação. Para Varshney \& Kumbhar (1978) a reologia, ou consistência de líquidos newtonianos semelhantes à água, leite ou suco de frutas clarificados, pode ser simplesmente caracterizada pelo termo viscosidade, com o que está de acordo Barnes et al. (1989), quando definem viscosidade como a conseqüência dos atritos internos na massa dos fluidos, representando resistência ao escoamento.

Face às considerações, estudaram-se as variações do teor de sólidos solúveis totais, $\mathrm{pH}$, vitamina $\mathrm{C}$ e viscosidade, em função do tempo de armazenamento e a caracterização reológica do suco de cinco genótipos armazenados em tubos plásticos e congelados a $-10^{\circ} \mathrm{C}$.

\section{MATERIAL E MÉTODOS}

Para obtenção do suco de acerola, coletou-se manualmente, em 02/10/98, $2 \mathrm{~kg}$ de frutas em fase fisiológica de maturação de cada um dos genótipos CL2, CL5, CL11, R1 e R2 oriundos do pomar da Faculdade de Ciências Agrárias e Veterinárias de Jaboticabal, SP. Após coletadas, as frutas foram lavadas, secadas com ajuda de toalha de algodão e esmagadas com processador manual, separando-se o suco e, ou, a polpa dos caroços, com auxílio de uma peneira (tela de $1,6 \mathrm{~mm}$ ); acondicionando-se $20 \mathrm{~mL}$ de suco em tubos plásticos $(5 \times 3 \mathrm{~cm})$. Posteriormente, esses tubos foram etiquetados e armazenados a $-10^{\circ} \mathrm{C}$ em freezer, pelo período de 10 meses.

As determinações de sólidos solúveis totais (leitura refratométrica - Pearson, 1973), $\mathrm{pH}$ (método potenciométrico - Lara et al., 1976), vitamina C (método da redução do iodo - Lara et al., 1976) e viscosidade aparente (viscosímetro de cilindros concêntricos LVDII+ da Brookfield) foram realizadas inicialmente e bimestralmente até o $10^{\circ}$ mês.

As medidas de viscosidade foram realizadas com registros dos dados de rotação (rpm); torque (\%); viscosidade aparente ( $\mathrm{Pa} \mathrm{s})$; tensão de cisalhamento $(\mathrm{Pa})$; taxa de deformação $\left(\mathrm{s}^{-1}\right)$; temperatura $\left({ }^{\circ} \mathrm{C}\right)$ e tempo $(\mathrm{s})$, com teoria explicada por Krieger \& Elrod em Bezerra (1997). Do conteúdo armazenado de $20 \mathrm{~mL}$, foram retiradas amostras de $10 \mathrm{~mL}$ de suco para mensurações, que processadas a uma temperatura constante de $25{ }^{\circ} \mathrm{C}$ numa taxa máxima de deformação de $264 \mathrm{~s}^{-1}$, resultaram em 12 valores correspondidos entre as variáveis acima analisadas. Foram plotados todos os pontos da taxa de deformação por tensão de cisalhamento e taxa de deformação por viscosidade aparente.

\section{RESULTADOS E DISCUSSÃO}

O comportamento do teor de sólidos solúveis totais ( $\left.{ }^{\circ} \mathrm{Brix}\right)$, $\mathrm{pH}$, vitamina $\mathrm{C}$ e viscosidade dos genótipos em função do tempo, encontra-se na Figura 1, onde se observa que viscosidade e vitamina $C$ foram os parâmetros que apresentaram as maiores variações para os diferentes genótipos e é notável a superioridade do genótipo CL-2 no teor de sólidos solúveis totais. Quanto ao $\mathrm{pH}$, todos os genótipos apresentaram leve tendência de declínio do $1^{\circ}$ ao $2^{\circ}$ bimestre, com aumento nos $4^{\circ}$ e $5^{\circ}$ bimestres, obtendo-se valores superiores aos da avaliação inicial.

Os genótipos R1 e R2 apresentaram os maiores teores de vitamina C. Constata-se ainda que, no genótipo R1, ocorre certa estabilidade pois, além de apresentar o maior teor de vitamina $\mathrm{C}$, esse mesmo teor só reduziu no $2^{\circ}$ bimestre, mantendo-se constante até o término das avaliações. Os demais genótipos apresentaram redução gradativa de vitamina $\mathrm{C}$, confirmando os resultados de Pimentel et al. (1996) e Sanches et al. (1996), quando afirmaram que ocorre redução gradativa no teor de ácido ascórbico da polpa de acerola armazenada a baixa temperatura.

Quanto à viscosidade aparente, nota-se, sumariamente, tendência de redução ao longo do tempo em todos os genótipos, além de grande dispersão das faixas genotípicas, podendo-se caracterizar os genótipos, segundo a viscosidade, pela sua capacidade de tempo de armazenamento. 

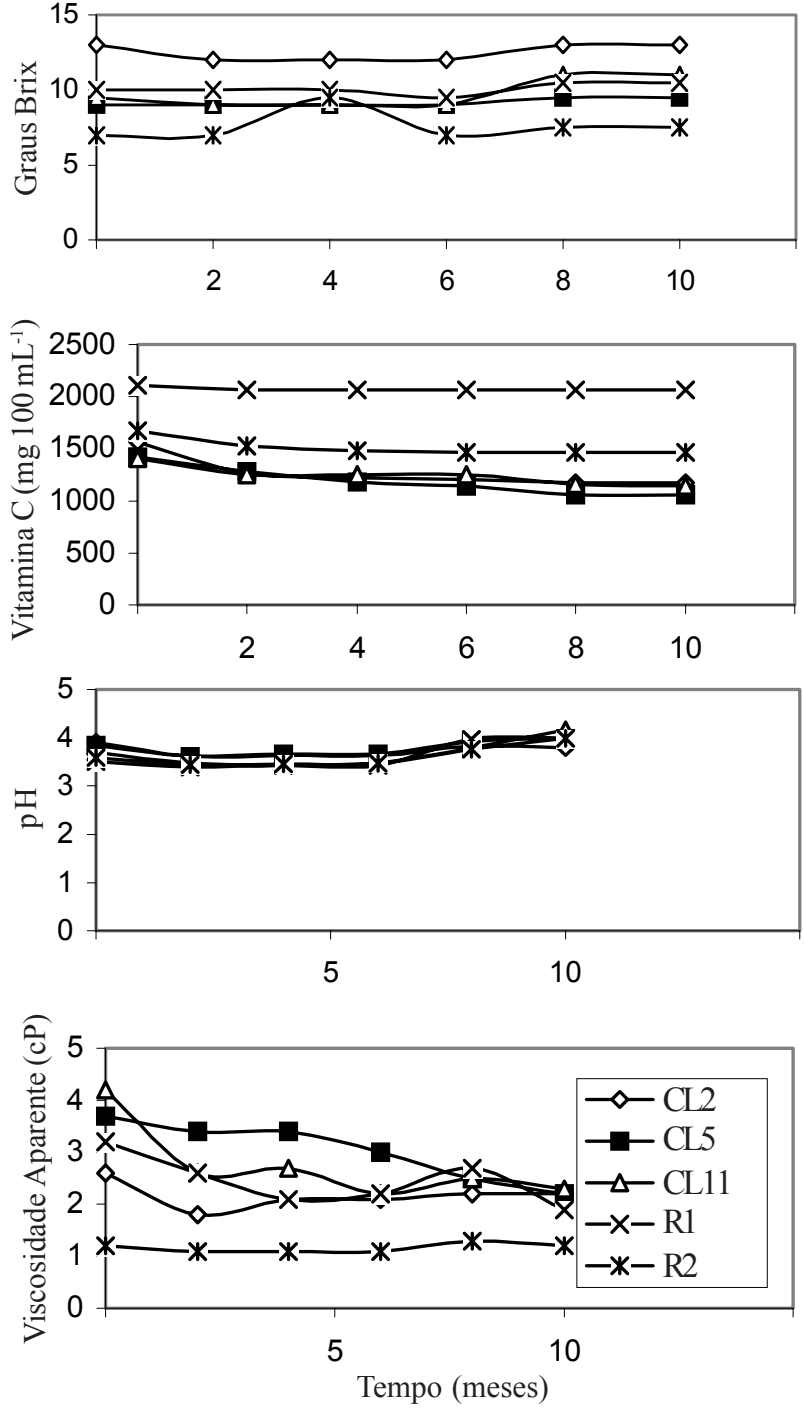

Figura 1. Variação no teor de sólidos solúveis totais ( ${ }^{\circ}$ Brix), $\mathrm{pH}$, vitamina $\mathrm{C}$ e viscosidade aparente em suco de acerola de cinco genótipos, sob temperatura de $-10^{\circ} \mathrm{C}$, num período de 10 meses

Na Figura 2 estão plotados os valores da tensão de cisalhamento pela taxa de deformação de cada genótipo ao longo do tempo. Os sucos dos cinco genótipos apresentaram em todas as avaliações bimestrais comportamento não-newtoniano mostrando inicialmente, crescimento gradativo entre tensão de cisalhamento e taxa de deformação até $250 \mathrm{~s}^{-1}$. Acima de $250 \mathrm{~s}^{-1}$ ocorreu certa estabilização de comportamento com tendência a tornar constante a tensão de cisalhamento, mesmo com aumento da taxa de deformação. Entretanto, algumas alterações são observadas no genótipo CL-11, indicando resposta genotípica específica.

A partir da taxa de $250 \mathrm{~s}^{-1}$, a tensão de cisalhamento torna-se praticamente constante, mesmo com crescimento da taxa de deformação, sugerindo que a deformação máxima do fluido ocorre no segundo mês de armazenamento, quando se verifica o maior distanciamento entre os valores da tensão de cisalhamento e taxa de deformação nos genótipos; portanto, o suco da acerola dos genótipos estudados não é caracterizado por uma relação linear entre tensão de cisalhamento e taxa de deformação, conforme descrevem Nagy et al. (1993) e seu
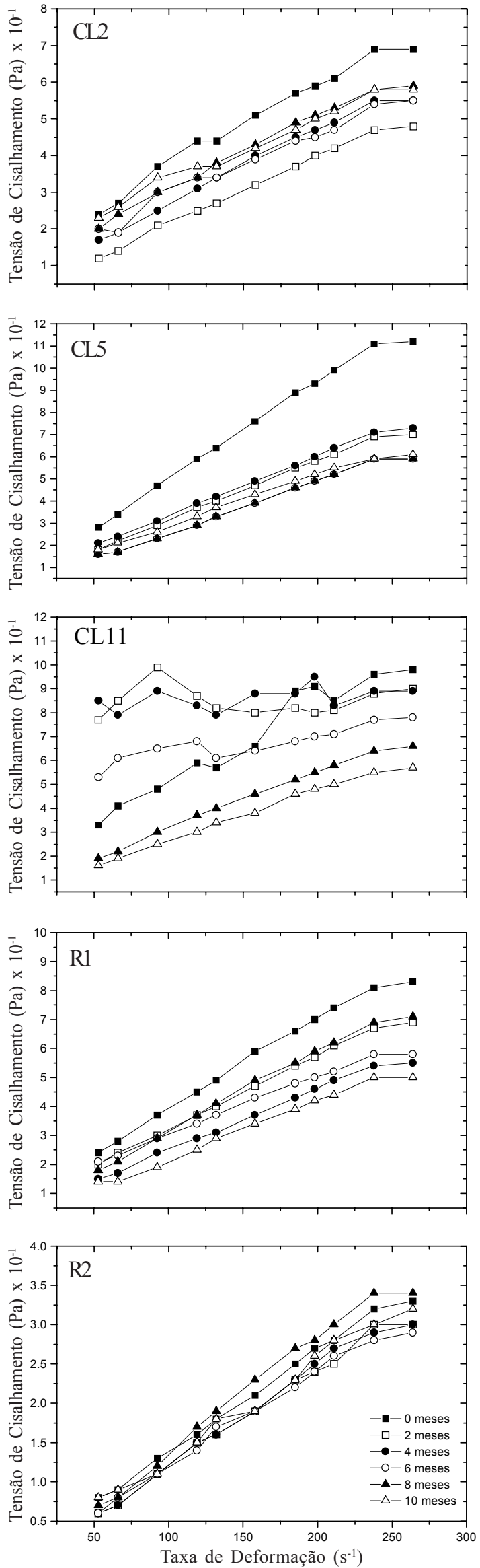

Figura 2. Relação entre tensão de cisalhamento e taxa de deformação para suco de acerola de cinco genótipos, armazenado em freezer $\mathrm{a}-10^{\circ} \mathrm{C}$, num período de 10 meses 

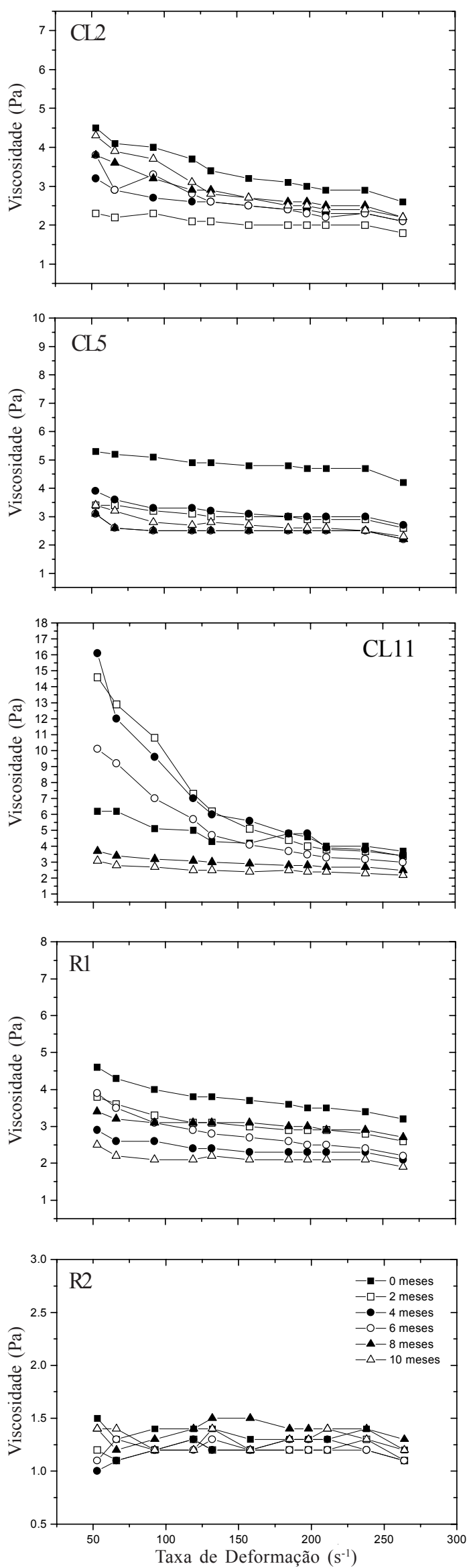

Figura 3. Relação entre viscosidade $(\mathrm{Pa})$ e taxa de deformação para suco de acerola de cinco genótipos, armazenados a temperatura de $-10^{\circ} \mathrm{C}$, num período 10 meses comportamento reológico pode ser utilizado como medida de qualidade (Ibarz et al., 1996), uma vez que a perda considerável de vitamina $\mathrm{C}$ ocorre somente no primeiro bimestre, mantendo, posteriormente, certa estabilidade (Figura 1).

Na Figura 3 visualiza-se uma relação entre viscosidade e taxa de deformação em suco de acerola armazenado em freezer, num período de 0 a 10 meses, demonstrando comportamento não-newtoniano e pseudoplástico (Trifiró et al., 1987) dos sucos estudados, visto que a viscosidade aparente decresce com o aumento da taxa de deformação, conforme relato de Holdsworth (1971); exceção feita ao genótipo R2, que apresenta pequenas oscilações da viscosidade até $200 \mathrm{~s}^{-1}$ nas avaliações inicial, do $1^{\circ}$ e do $2^{\circ}$ bimestres, a partir do que, a viscosidade tende a se tornar constante; por outro lado, nas determinações dos $3^{\circ}, 4^{\circ}$ e $5^{\circ}$ bimestres, a redução da viscosidade é mínima, constatando-se manutenção parcial da viscosidade aparente, podendo caracterizar-se o fato como resposta particularizada do genótipo, ao longo do tempo.

Conforme sugestão apresentada no texto, analisando o comportamento do R2 em relação aos demais genótipos, não se observa redução gradativa da viscosidade com o aumento da taxa de deformação, demonstrando pequena modificação no comportamento reológico; fato justificado, pois os derivados de frutas são sistemas bifásicos compostos por partículas sólidas dispersas, com distribuição e conformação estrutural ao longo do tempo de armazenamento (Costell \& Durán, 1982) e, que, segundo Trifiró et al. (1987) seu comportamento reológico pode alterar-se por ação enzimática ou mecânica que modifique a estrutura da polpa e ou mesmo que tal fato seja característica genotípica específica. Sobre o tema, Tanglertpaibul \& Rao (1987) reportaram que o comportamento reológico de sucos e purês de frutas está ligado aos sólidos solúveis em suspensão em função da forma, tamanho, concentração das partículas suspensas e da estrutura do sistema; fenômeno que pode ser confirmado pela análise de variação do teor de sólidos solúveis totais (Figura 1) e, pela visualização de partículas suspensas durante a avaliação do último bimestre, quando se notou pequena quantidade dessas partículas no genótipo R2 e maior quantidade delas nos demais; com isso, reforça-se também a afirmação anterior de Trifiró et al. (1987).

Considerando-se a taxa de deformação na Figura 3, observa-se que, ao longo do tempo, ocorreu redução de viscosidade nos sucos dos genótipos, verificando-se certa rapidez no primeiro bimestre ( 2 meses), moderadamente lenta do segundo (4 meses) ao terceiro bimestre (6 meses) e muito lenta do quarto ( 8 meses) ao quinto bimestre (10 meses) e, conseqüentemente, reduziu-se também o atrito na massa dos fluidos, apresentando, portanto, menor resistência ao escoamento sugerindo, desta maneira, um envelhecimento gradativo do derivado.

\section{CONCLUSÕES}

Considerando-se as condições em que se realizaram os experimentos, conclui-se que:

1. A viscosidade, seguida da vitamina $C$, apresentaram as maiores variações nos genótipos.

2. O genótipo CL-2 apresentou superioridade no valor de sólidos solúveis totais. 
3. Os genótipos R1 e R2 apresentaram maior teor de vitamina $\mathrm{C}$.

4. Os sucos dos genótipos estudados apresentaram comportamento não-newtoniano e pseudoplástico.

5. As maiores modificações dos sucos ocorreram nos dois primeiros meses.

\section{AGRADECIMENTOS}

À FAPESP, pela concessão de bolsa e apoio financeiro ao projeto.

\section{LITERATURA CITADA}

Barnes, H.A.; Hutton, J.F.; Walters, K. An introduction to rheology. New York: Elsevier Science Publishers, 1989. 189p.

Bezerra, J.R.M.V. Estudo reológico do suco de manga. efeito dos sólidos insolúveis. Campinas: FEAGRI, UNICAMP. 1997, 81p. Dissertação Mestrado

Costell, E.; Durán, L. Reologia y fisico-química de los zumos y purés de frutas. Revista de Agroquimica y Tecnologia de Alimentos, Valencia, v.22, n.1, p.81-94, 1982.

Holdsworth, S.D. Applicability of rheological models to the interpretation of flow and processing behavior of fluid food products. Journal of Texture Studies, Chicago, v.2, n.4, p.393-418, 1971.

Ibarz, A.; Gonzals, C.; Esplugas, S. Rheology of clarified passion fruit juice. Fruit Processing, Chicago, v.6, n.8, p.330-333, 1996.

Lara, A.B.W.; Nazário, G.; Almeida, M.E.W.; Pregnolato, W. Normas Analíticas do Instituto Adolfo Lutz - Métodos químicos e físicos para análise de alimentos. São Paulo: Instituto Adolfo Lutz, n.1, 2 ed., 1976.
Nagy, S.; Chen, C.S.; Shaw, P.E. Fruit juice processing technology. Miami: Agscince Ins.; Albuquerque, 1993. $713 \mathrm{p}$.

Pearson, D. Laboratory techniques in food analysis. London: London Butterwoths, 1973, p.58-60.

Pimentel, M.L.; Maia, G.A.; Oliveira, G.S.F.; Silva Jr., A. Influência do processamento sobre a vitamina $\mathrm{C}$ do suco de acerola. In: Congresso Brasileiro de Ciência e Tecnologia de Alimentos, 15, 1996, Poços de Caldas. Resumo... Poços de Caldas, 1996. p.266.

Queiroz, A.J. de M. Estudo do comportamento reológico dos sucos de abacaxi e manga. Campinas: FEAGRI, UNICAMP. 1998. 170p. Tese Doutorado.

Rao, M.A.; Anantheswaram, R.C. Rheology of fluids in food processing. Food Technology, Chicago, v.36, n.2, p.11626, 1982.

Sanches, J.; Kanesiro, M.A.B.; Durigan, J.F.; Tostes, D.R.D.; Kanesiro, J.C. Qualidade da polpa de acerola congelada. In: Congresso Brasileiro de Ciência e Tecnologia de Alimentos, 15, 1996, Poços de Caldas. Resumos... Poços de Caldas, 1996. p.134-135.

Tanglertpaibul, T.; Rao, M.A. Rheological properties of tomato concentrates as affected by particle size and methods of concentration. Journal of Food Science, Chicago, v.52, n.1, p.141-145, 1987.

Trifiró, A.; Saccani, G.; Gherardi, S.; Bigliardi, D. Effect of content and sizes of suspended particles on the rheological behaviour of apricot purees. Industria conserve, Chicago, v.62, p.97-104, 1987.

Varshney, N.N.; Kumbhar, B.K. Effect of concentration and temperature on rheological properties of pineapple and orange juices. Journal of Food Science Technology, Oxford, v.15, p.53-55, 1978 . 